\title{
Twinning with Tonga: The Experiences of Tongan Stakeholders with a Long-term Partnership with Regional Victoria, Australia
}

\author{
Carolyn Mornane $^{1, *}$, Michelle Franc ${ }^{2}$, Maureen Waddington ${ }^{3}$, Blake Peck ${ }^{4}$, Daniel Terry ${ }^{4}$ \\ ${ }^{1}$ Clinical Nurse Consultant Stomal Therapy, St John of God Hospital, Australia \\ ${ }^{2}$ Manager of Health Information Services, St John of God Hospital, Australia \\ ${ }^{3}$ Director of Mission, St John of God Hospital, Australia \\ ${ }^{4}$ School of Nursing \& Healthcare Professions, Federation University Australia, Australia
}

Copyright $\bigcirc 2019$ by authors, all rights reserved. Authors agree that this article remains permanently open access under the terms of the Creative Commons Attribution License 4.0 International License

\begin{abstract}
Twinning programs in health have gained increased recognition as a WHO preferred strategy for providing a sustainable strategy for enhancing the delivery of best practice healthcare globally. The Tonga Twinning Program (TTP), represents a longstanding relationship of some twenty-five years between The Ministry of Health in Tonga and St John of God Hospital, Ballarat, Australia and provides a compelling example of what can be achieved. This article presents the findings from a longitudinal exploration of the experiences and perceptions of the TTP through the voices of those key-stakeholders situated in Tonga who have engaged with the program. Informed by the tenets of hermeneutic phenomenology, a modified thematic analysis highlighted two major themes, 'A shared mission' and 'The outcomes are more than the tangibles', which supported by a series of sub-themes, identify the core components of the experience of the TTP. This study suggests that the TTP has supported a collective sense of bringing the very best available knowledge and skills to the people of Tonga and has fostered a genuine and open dialogue between partners as a mechanism for change that goes well beyond simply a capacity to replicate skills and instead has establish a genuine reciprocity akin to being a family.
\end{abstract}

Keywords Twinning, Healthcare Partnerships, Qualitative, Tonga

\section{Introduction}

This paper contributes to the developing body of evidence for Twinning in healthcare where a collaborative relationship is established between two organisations that are similar in some way (Hong, et. al., 2012). Specifically, this study focuses on the experiences and outcomes the Tonga Twinning Program (TTP), an international collaboration between St John of God Ballarat Hospital, (SJGBH) Australia, and the Ministry of Health, Tonga, (MOHT). A close examination of the experiences and perceptions of the TTP through the voices of key-stakeholders from diverse areas, both Clinical and Non Clinical provides insight into the widespread and long-term impact Twinning programs can make.

In an era of ever increasing disparity in access to resources, achieving goals like that of the United Nations, which seek to 'Ensure health lives and promote wellbeing for all at all ages' by 2030, a variety of collaborative approaches are required. Twinning is considered one of the possible approaches [1-3]. Twinning in a healthcare context is an approach that brings together health professionals from different countries and cultures in an effort to improve healthcare of both countries through the empowerment of both visiting and welcoming healthcare professional [4-5].

Advocacy for Twinning approaches is strong with the Swedish International Development Co-operation Agency (SIDA) where Jones and Blunt [6] describe Twinning as having advantages over other forms of development co-operation. Such an approach encourages sustainable and deeper levels of organisational engagement with stronger ties between countries. Echoing this position, Collins, Burn and Ritman [7] suggest Twinning provides a flexible framework for healthcare professionals and organisations to work effectively and responsively for longer periods than other typical approaches. In addition, it provides continuity and strategic mobility beyond the life-cycle of any single small-scale project, and in so doing provides impetus for lasting relationships and longer term outcomes.

In terms of bringing together health professionals across 
cultures in an effort to improve healthcare by empowering these professionals [8], Twinning does appear to have great potential for this purpose [1]. Despite this, SIDA suggests that this potential has not yet been fully realised and exploited globally [9]. One reason for this is that Twinning programs have been implemented in a variety of ways that span the spectrum of straight forward linking of organisations in an effort to share established resources including policies and procedures to more complex arrangements that may or may-not include periods of exchange visits in order to achieve a complex bilateral developments between healthcare organisations.

While this breadth is strength, Cadee, et.al [1] argue that "without definitional clarity and a full understanding of the concept, it is difficult to assess the value of Twinning or to discover which aspects of Twinning contribute to its success" (p. 2). In an effort to establish a common foundation, a definition, of Twinning programs from which to then be able to make determinations of a program's effectiveness, Cadee, et.al.[1], engaged in a content analysis of existing literature to define the key attributes of Twinning in international health collaboration.

Cadee, et.al [1] arrived at the following definition, "Twinning is a cross-cultural reciprocal process where two groups of people work together to achieve joint goals." (p.8). Within this process four key attributes are consider dot exits that ensure the effectiveness of Twinning, including: reciprocity, building personal relationships, dynamic process, and participation of two organisations across cultures. Cadee et.al., [1] further indicate the core of effective Twinning is the establishment and maintenance of genuine reciprocity, whereby each party is empowered to play their role within the relationship. Building strong personal relationships supports an openness that sustains this reciprocity and provides a sense of empowerment to make spontaneous or dynamic decisions about a particular focus of attention [10-11]. In so doing, the relationship is one of partnership over dependence which leads to the empowering effect of Twinning.

Successful Twinning has the capacity to empower healthcare professions across the world to influence and make positive change [10]. However, Jones and Blunt [5] suggest that the strengths of Twinning are in need of further scientific studies to underpin assumptions of effectiveness [1]. This paper contributes to the developing body of knowledge regarding the effectiveness of Twinning programs by examining of the long-standing Twinning program between St John of God Hospital (SJoG) and the Ministry of Health, Tonga, (MOHT). To appreciate the depth of this partnership an introduction of the Tong Twinning Program (TTP) is useful.

\subsection{Objectives}

In 2017, the TTP between SJOGBH and Vaiola Hospital, Tongatapu (VHT) celebrated its $25^{\text {th }}$ Anniversary. The program functioned under shared funding from the
Australian Government and SJOG. The relationship involves the reciprocal exchange of staff between SJOGBH, Australia, and MOHT. Since its inception more than 120 Tongan Ministry of Health employees have experienced one-month placements twice a year in Ballarat developing capacity across all aspects of health care. Despite the similarity in hospital values, staff from SJOGBH who visited Tonga witnessed the strong sense of inclusion, including being welcomed into their culture. As a function of this, SJOGB staff has been able to return to the core values of the institution by way of a welcoming program that is delivered to each delegation of Tongan visitors emphasising the history of StJoGB and the shared values of the Twinining organisations. Reciprocal visits of two-weeks twice a year have been made to Tonga by more than 60 SJOGBH staff members who have, through their experience, developed an appreciation and admiration for the skills of the care providers in the under resourced Tonga health service. While the longevity of the program is in some way a measure of its success, a long-term qualitative exploration of the experiences and perceptions regarding the TTP demonstrates its effectiveness.

\section{Methods}

Interpretative phenomenology informed by the philosophy of Hans-Georg Gadamer[12] (1960) was utilised to underpin the qualitative component of the study. The ontological nature of the philosophy enabled an exploration of the everyday meanings that people constructed of their realities with and of the Twinning program [13]. Moreover, the hermeneutic phenomenological approach enabled the essence of the experience to be explored through the words of those people immersed within the phenomena of Twinning itself [12].

Following ethical approval from SJOGBH and MOHT a purposive sampling technique was employed to identify staff from all areas to take part in one-one semi-structured interviews. We identified a key senior stakeholder to assist us in gaining participation to assist us with available participants for all key areas. It became apparent that stakeholders across all disciplines (Clinical and Non Clinical) outside of the nursing discipline were enthusiastic about sharing their experiences of the TTP and we engaged a snowball sampling technique to recruit the remainder of the final sample. Participants were interviewed in Tonga during in-country visit by SJOGBH staff. In total 33 people were interviewed with a mix of both clinical and non-clinical roles from all areas of the Ministry of Health, Vaiola Hospital Tongs. Interviews were digitally recorded and later transcribed by the research for analysis.

The technique for phenomenological data analysis (verbatim transcription, extraction of significant statements, identify similarities in formulated meanings, group the 
similar meanings, create an exhaustive statement) proposed by Collaizi [14] was used for thematic development in order to uncover the hidden and ambiguous nature of the experiences of key-stakeholders interviewed. In-depth contact with the transcripts and the audio recordings was maintained and support the identification of significant statements that pertained to the phenomena of interest. The meanings formulated were then clustered together to form themes that encompassed the experiences of Tongan people engaged with the Twinning project.

Rigour was ensured in several ways. Although the researchers who conducted the interviews prepared the transcripts, other members of the research team so as to ensure accuracy of the data for analysis again compared the transcripts with the audio-recordings. Given English as a second language of the Tongan people who shared their experiences, it was considered culturally inappropriate to return the transcripts to the participants [15]. Instead, at each stage phase of data analysis the emergent themes and their descriptions were cross checked between members of the research team.

The outcomes of this process of analysis are a series of two major themes: A shared mission and the Outcomes are more than merely the tangibles. The former has two sub-themes: Achieving the mission and Mission barriers. In combination these themes come to represent the experiences of those Tongan people who engaged with the TTP.

\section{Findings}

The experience of the people from Tonga who engaged with the TTP can be broadly clustered under the major theme A shared mission which is then amplified by a series of three sub-themes: Achieving the mission, Mission barriers, and The outcomes are more than merely the tangibles, each of these is unpacked in detail below.

\subsection{A Shared Mission}

Participants emphasised that during the initial stages of the programs implementation it became evident that the connection with SJGBH was more than merely 'another international partnership'. Instead of a sharp focus upon a predetermined end goal, participants described a strong sense of the importance of having shared values between Vaiola Hospital and StJoGB. The term 'mission' here represents not only the shared religious faith, it also represents the journey of shared achievement. This sentiment - while widely shared by the participants - is exemplified by the following:

We are so happy not only with the hospital in Australia, but the kind of principles that are in place there. I think that is the difference. You can have a partnership with any hospital then to have a partnership with a hospital that has those principles is very important to us. I think that is why we support its concept and I think it is great. (NC Management)

Embedded also in the theme itself in relation to the shared values is a recognition that the involvement of the people from Tonga was part of something, part of a 'mission' to bring the best international standards to Tonga in order to continue to be the very best they can be.

The Twinning program is really helpful for our setting here....To be the best and be judged by international standards, We continue to have this Twinning program it continues to push us to work towards what we have to achieve. (Clinical Nursing)

We learn from Ballarat with continuous improvement and there is hope for everything with the positive and enthusiasm of your team. (Clinical Nurse)

It's for the country (Clinical Nursing)

It is evident that the experience of Twinning for those from Vaiola is more than merely the achievement of focused goal in effort to meet the needs of a funding body. Instead, it represents something deeper for the partners. There is a genuine sense that being involved in the Twinning program is developing the present and future health opportunities for the people of Tonga.

\subsection{Achieving the Mission}

Participants described the role that making the 'right' selection of staff from Vaiola Hospital to come to the StJoGB site as being central to the overall and ongoing success of TTP program. Participants described having at least two people attend the StJoGB site from a specific clinical or non-clinical setting in Vaiola Hospital helped to consolidate and implement practice.

Two people helping each other, trying to push very hard to prove themselves... for the best of the people of Tonga. If there are two people they can support each other in that change. They can share their idea and help each other and encourage each other for the better change in Tonga. (Clinical Nursing)

[We need to] pick wisely who comes from Vaiola Hospital Two from each section. Help each other, need skills for improvement of services. Someone to support times-two can share ideas of what they have to look for better change. (Clinical Nursing)

As well as comments about the number of people, participants also highlighted their belief that the ability of this specific TTP program to be inclusive of individuals from multiple settings was critical to its long-term success.

What is good is that you take people from various sections, administration, [and] nursing. Now we are sending people in Information Technology, Medical Records, so they continue to grow (Non-Clinical Management) 
Participants expanded on this point and identified that the engagement of staff from various clinical and non-clinical areas worked effectively to establish a change in the workplace culture that was sustained by their shared experiences and vision.

It changes the atmosphere and makes everyone feel at home and makes an environment to learn (Clinical Nursing)

I think exposure to everyone will be better for the development of Staff from Vaiola Hospital (Clinical Nursing).

Central to the longstanding success of the TTP program is the ability of the partners to identify staff/settings that are the best fit for opening up opportunities for developing Vaiola Hospital staff visiting the StJoGB site. This breadth of the TTP is evidenced by those clinical and non-clinical services that were selected and the subsequent depth of the TTP is strongly supported by the recommendation that two people are sent from each area. In combination, these elements are considered by participants to be supportive of achieving the mission of the TTP program.

\subsection{Mission Barriers}

Participants expressed what they perceived to be aspects of the TTP program that operate as barriers to the overall success of achieving the mission to improve the provision of healthcare for the people of Tonga. One of the strongest themes that emerged from the data centred on ensuring that people who had been part of the TTP program were provided with sufficient 'longevity' in the position for which they had received the extended support and development from the StJoGB team. The following quote is indicative of the perceptions of the wider participant group.

That's a part of the big challenge when Tongan staff get moved to another area after they have been to Ballarat and it is out of our control (Clinical Nursing)

At the centre of this wish to keep staff who have experienced StJoGB is a desire to build capacity amongst the rest of the team at Vaiola Hospital a positioned well captured by the following:

Need to make sure people stay in that area and build up the capacity (Clinical Nursing)

This theme captures the way in which participants in the TTP program developed a strong sense of the importance of having a strategy for maintaining the newly developed knowledge, skills and their application within a clinical or non-clinical area for sufficient time so as to change or influence current practice within that area. In so doing, this strategy helps to build capacity amongst the members of the other members of the designated area/setting. The 'out of control' nature of the health professionals - clinical and non-clinical - shifting between different areas within the organisation soon after having visited the StJoGB site was considered an impediment to the success of the program. Instead, what is recommended is that people who have engaged in the exchange visit are provided with support to remain in their area of expertise, or speciality for a period of time upon their return.

\subsection{The Outcomes are more than the Tangibles}

Participants described the outcomes of the TTP in different ways and tended to reflect on the entire period or mission of the collaboration. There were reflections of the more tangible skills and practices that have been acquired and refined over the period of the mission.

"The benefits are that is helps our staff to get more skills and knowledge in patient management also communication with clients and also how the general communication in the hospital". (Non-Clinical Management)

"Time management and staff management, culture environment and staffing and how they recruit the new staff." (Non- Clinical Management)

However, the data would suggest that at the heart of the outcomes achieved by the TTP is a strong sense of the 'genuineness' of the relationship. Here the TTPs ability achieve the mantra of the United Nations to 'Ensure healthy lives and promote wellbeing for all ages - 2030' becomes evident. With better and more genuine relationships there comes a subsequent ease of communication where learning is enhanced and is arguably the most important long-term non-tangible outcome. Undoubtedly a relationship is a factor in the overall success of the TTP, however, it is elaborated in this theme in terms of an outcome in recognition of the genuine nature of the relationship that has been articulated. Participants routinely describe the way that they felt that the TTP team from StJoGB were like family and could communicate with ease.

"StJoGB just took us like part of the family" (Clinical Nursing)

"What works is the collaboration sharing of knowledge between us...we email them, they email us" (Clinical Nursing)

"We have built relationships which continue to help us in Tonga" (Non-Clinical Management)

In culmination this sense of the empowerment that comes from a genuine relationship with their StJoGB counterparts created a greater sense or recognition amongst the people at Vaiola that the most important component of any workplace is the creation and maintenance of good relationships.

It is important with the good relationship between everyone in every section (Clinical Nursing)

This theme embodies the idea that the outcome of a successful program such as the TTP with StJoGB are more than merely the tangible elements of skills, practices or 
processes that shape the way an intuition such as Vaiola Hospital runs. Instead, this theme recognises that the most central outcome of the TTP is the genuine and 'easy' relationship between the people of the sister institutions. This ease is reflected by the use of the word 'family' where one feels secure to engage in non-judgmental dialogue that is ultimately seen as providing a better experience for partners.

\section{Discussion}

The findings represent a long-term exploration of the experiences and perceptions of the effectiveness of the TTP from the perspective of the Tongan people by way of the shared voices of those key-stakeholders immersed in the program itself. Interestingly, there is very little literature that describes - in their own words - the experiences of being involved in a Twinning program itself. Given this, the themes emerging from the data are situated within the existing and wider literature by way of the work of Cadee et.al.[1], specifically the four key attributes of Twinning outlined above.

The attribute of reciprocity, or the exchange of things with others for mutual benefit, is embodied across the themes identified here, particularly, within the theme of ' $A$ Shared Mission'. It was evident from the findings that key-stakeholders within the partnership where able to establish and maintain an open and genuine dialogue throughout the mission and to establish a mutual respect for the position of each partner over time as argued by Collins, Burn and Ritman [6]. In so doing, the TTP was able to instil a deep sense of mutual ownership of the program and the direction that was taken, a position supported by Jones and Blunt [5]. This notion of being spontaneous is supported by Cadee's et.al. [1] suggestion that genuine Twinning program are dynamic in nature.

Having people from both clinical and non-clinical areas of the health-service partners meant that the program was able to be dynamic with regard to the exchange of learning that and made the TTP a dynamic experience. Arguably, at the heart of a dynamic Twinning program is the establishment of close personal relationships. The findings from this study suggest that having established a foundation of open and genuine dialogue 'akin to a family', facilitated the partners reaching agreement about the direction to be taken. This is certainly supported by literature that takes a focus on Twinning program in midwifery where researchers have described the relationships in terms of sisters [1-2, 8, 16-18]. While the experiences of those people engaged with the TTP outlined here reflect the key criteria for a genuine Twinning program as outlined by Cadee's et.al. [1], the findings offer further nuanced understanding about those factors that have contributed to the longevity and ongoing success of the TTP program outlined here.
While other authors have focused on the tangible outcomes of the specific Twinning programs with which they have been involved [7, 19], there is only minimal discussion within the literature that could be considered to explore - the arguably greater - value of the non-tangible outcomes. There are authors who describe the close personal nature of the relationships that have been formed (8, 16-18, 20-21) and their importance to a programs longevity[7] but they stop short of articulating the implications of this 'comfort' or 'ease' created within the relationships as the foundation for empowerment. In the TTP participants highlighted a sense of equity in the program having engaged both non-clinical as well as clinical staff. This is perceived as a very positive mechanism for establishing a neutral-power relationship like a family - that fosters open dialogue and unity. In so doing, the relationship formed is not founded on dependence but on empowerment of both parties.

The findings from this study suggest that in addition to establishing a neutral power relationship, the inclusion of both non-clinical and clinical staff, was recognized as a key factor in the success and longevity of the TTP and is a contribution that is a seemingly new development within the literature. The WHO [22] articulate how successful Twinning partnerships enhance institutional capacity to deliver improved health services. We would suggest that having two people from each area/setting/unit participate in the program at the same time has been a positive strategy for promoting and maintaining a cultural of ongoing learning and dissemination of ideas amongst the wider area of practice. This finding represents a development in our understanding of Twinning programs that offers a recommendation for future Twinning programs seeking to increase the sustainability of the learning. Consistent with this, participants identified that where those people who had engaged in the TTP were moved out of the area - both non-clinical and clinical - for which they had received specific development/training or engagement, the ongoing learning and broader cultural shifts were reported as being poorly sustained. Again, this finding provides a recommendation that a process of forward planning is undertaken to ensure that the expertise are retained for a period of time at the completion of a period of engagement with the other respective partner organisation.

\section{Conclusions}

The findings from this study represent a qualitative exploration of the experiences and perceptions of the effectiveness of the TTP from the Tongan perspective through the shared voices of those key-stakeholders immersed in the program itself.

The findings from the long-term engagement between the two organisations -Vaiola and StJoGB - are overwhelmingly positive about the outcomes of the TTP. 
The TTP has been described as not only providing a platform for evolving knowledge, skills and their application in a variety of settings - or hard skills - but also providing the foundation for the refinement of 'softer skills'. Evidence of these 'softer skills' can be seen by the 'inner drive' that participants described in relation to their recognition that the TTP and their involvement in it was for the people of Tonga, for the country.

This powerful realisation is a significant outcome that arguably stems from the close, personal relationships that have been forged between the two partners and their respective institutions, providing a sense of openness to dialogue that ultimately disentangles dependence and fosters empowerment. In culmination we argue that the TTP established between the two parties - Vaiola and StJoGB - exceeds the standards set of previous research with regard to what constitutes an effective Twinning program. The findings identified here suggest that future research might explore the perceptions of those engaged in similar Twinning programs in order to evaluate the value of having two staff from each area as well as forward planning in order to maximise the contact of personnel within their specific area of development.

\section{Acknowledgements}

We are very grateful to Pinomi Latu for assisting with the recruitment of participants for this study as well as the ongoing support of the Ministry of Health, Tonga.

\section{REFERENCES}

[1] F. Cadee, M.J., Nieuwenhuijze, A. Lagro-Janssen, \& R. De Vries. The state of the art of Twinning, a concept analysis of Twinning in healthcare. Globalization and Health, 12(1), 66, 2016.

[2] F. Cadee, M.J., Nieuwenhuijze, A. Lagro-Janssen, \& R. De Vries. From equity to power: Critical Success Factors for Twinning between midwives, a Delphi study. Jounal of Advanaced Nursing, 74, 1573-1582, 2018.

[3] UN. United Nations Sustainable Development Goals. 2015. Retrieved from https://sustainabledevelopment.un.org/topi CS

[4] E. Ndenga, G. Uwizeye, D.R. Thomson, E. Uwitonze, J. Mubiligi, B.L. Hedt-Gauthier, et al. Assessing the Twinning model in the Rwandan human resources for health program: goal setting, satisfaction and perceived skill transfer. Glob Health. 12:4, 2016.

[5] R. Sandwell, D. Bonser, E. Hebert, K. Kilroy, S. Leshabari, F. Mwanga, A. Mtawa, A. Wilson, \& A. Moritz, Stronger together: midwifery Twinning between Tanzania and Canada, Globalization and Health 14:123, 2018.

[6] M. Jones, P. Blunt. 'Twinning' as a method of sustainable institutional capacity building. Public Administration and Development, 19, 381-401, 2009.

[7] M. Collins, E. Burn, D. Ritman. A partnership model for the training and professional development of health-care staff in low-resource settings. Cancer Control, 79-86, 2015. Retrieved from www.cancercontrol.info/wp-content/uploa ds/2015/07/79-86-Collins.pdf

[8] ICM. International Confederation of Midwives Twinning manual. In: Moyo NT, editor. B.M.A.; 2014. http://www.internationalmidwives.org/assets/uploads/docu ments/Twinning/140419\%20Twinning\%20ICM\%20V04.p df. Accessed 15 Oct 2016.

[9] P.E. Farmer, \& J.Y. Kim, Surgery and global health: a view from beyond the OR. World Journal of Surgery, 32(4), 533-536, 2008.

[10] F. Adloff, \& S. Mau. Giving social ties, reciprocity in modern society. Eur J Sociol. 47(01):93-123, 2006.

[11] N. Wallerstein. Empowerment to reduce health disparities. Scandi navian Journal of Public Health, 30(59 suppl), 72 77, 2002. https://doi.org/ 10.1177/14034948020300031201

[12] H.G. Gadamer. Truth and method. New York: Continuum Press. 2003. (Original work published 1960)

[13] N. Denzin, \& Y. Lincoln. The Sage Handbook of Qualitative Research ( $5^{\text {th }}$ Ed.). London: SAGE Publishing. 2017.

[14] P. Colaizzi. Psychological research as the phenomenologisl views it. In Valle, R. and King. M. (eds.). Existential phenomenologica/ alternatives for psychology. New York: Ox.ford University Press. 1978.

[15] H.P. Santos, A. M. Black, \& M. Sandelowski. Timing of translation in cross-language qualitative research. Qual Health Res, 25:134-44, 2015.doi:10.1177/1049732314549 603

[16] K. P. Herschderfer. Assessment of the KNOV-SLMA Twin-to Twin Program Perceptions and experiences of twinned midwives and lessons learned. Amsterdam: Royal Tropical Institute, KIT. 2012

[17] RCM, Royal College of Midwives. Global midwifery Twinning project. 2015

[18] F. Cadée. 'Twin2twin' an innovative method of empowering midwives to strengthen their professional midwifery organisations. Midwifery. 29(10):1145-50, 2013.

[19] J. Hopkins, E. Burns, \& T. Eden. International Twinning partnerships: An effective method of improving diagnosis, treatment and care for children with cancer in low-middle income countries. J Cancer Policy. 1 (1):e8-19, 2013.

[20] S. Breiddal. The How-to Guide to Hospice Palliative Care Twinning Projects Health Canada, Editor. 2009.

[21] H. Jiang, J. Ives Erickson, M. Ditomassi, \& J.M.Adams. Promoting a Culture of International Professional Practice for Nursing through a Twinning Relationship. JONA: The Journal of Nursing Administration, 42 (2), 117-122, 2012. doi: 10.1097/NNA.0b013e318243384e.

[22] WHO. Partnership Preparation Package - a practical guide 
to implementing Twinning partnerships. Geneva: World Health Organization, 2018 (WHO/HIS/SDS/2018.13).

Licence: CC BY-NC-SA 3.0 IGO

[23] W. Zabierowski, A. Napieralski. Chords classification in tonal music, Journal of Environment Studies, Vol.10, No.5, 50-53.

[24] A. Abiewskiro, Z. Moplskiiera. The Problem of Grammar Choice for Verification, TCSET of the International Conference, House of Lviv Polytechnic National University, 19-23, 2008.

[25] Farquhar C, Protein and DNA Music, Online available from http://www.hrpub.org 